\title{
Anesthesiologists in Times of Disaster
}

Editors

JESSE RAITEN

LEE A. FLEISHER

\section{ANESTHESIOLOGY CLINICS}

www.anesthesiology.theclinics.com

Consulting Editor

LEE A. FLEISHER

June 2021 • Volume 39 • Number 2 